\title{
Successful renal transplantation following prior bone marrow transplantation in pediatric patients
}

Thomas SE, Hutchinson RJ, DebRoy M, Magee JC. Successful renal transplantation following prior bone marrow transplantation in pediatric patients.

Pediatr Transplantation 2004: 8: 507-512. @ 2004 Blackwell Munksgaard

Abstract: Improving survival rates following pediatric bone marrow transplantation (BMT) will likely result in greater numbers of children progressing to end-stage renal disease (ESRD) because of prior chemotherapy, irradiation, sepsis, and exposure to nephrotoxic agents. Renal transplantation remains the treatment of choice for ESRD; however, the safety of renal transplantation in this unique population is not well established. We report our experience with living related renal transplantation in three pediatric patients with ESRD following prior BMT. Two patients with neuroblastoma and ESRD because of BMT nephropathy, and one patient with Schimke immuno-osseous dysplasia and ESRD because of immune complex mediated glomerulonephritis and nephrotic syndrome. Age at time of BMT ranged from 2 to 7 yr. All patients had stable bone marrow function prior to renal transplantation. Age at renal transplant ranged from 8 to $14 \mathrm{yr}$. All three patients have been managed with conventional immunosuppression, as no patient received a kidney and BMT from the same donor source. These patients are currently 7 months to $6 \mathrm{yr}$ status post-living related transplant. All have functioning bone marrow and kidney transplants, with serum creatinine levels ranging $0.6-1.2 \mathrm{mg} / \mathrm{dL}$. There have been no episodes of rejection. One patient with a history of grade III skin and grade IV gastrointestinal-graft-vs.-host disease (GI-GVHD) prior to transplantation, had a mild flare of GI-GVHD (grade I) post-renal transplant and is currently asymptomatic. The incidence of opportunistic infection has been comparable with our pediatric renal transplant population without prior BMT. One patient was treated for basal cell carcinoma via wide local excision. Renal transplantation is an excellent option for the treatment of pediatric patients with ESRD following BMT. Short-term results in this small population show promising patient and graft survival, however long-term follow-up is needed. Pre-existing immune system impairment and bone marrow function should be taken into consideration when weighing different immunosuppressive agents for renal transplantation. Patients who have undergone renal transplantation following BMT are at high risk for opportunistic infections and malignancy, and need life-long medical surveillance.

\section{Susan E. Thomas', Raymond J. Hutchinson ${ }^{2}$, Meelie DebRoy ${ }^{3}$ and John C. Magee ${ }^{3}$}

Sections of ${ }^{1}$ Pediatric Nephrology, ${ }^{2}$ Pediatric Blood and Marrow Stem Cell Transplantation,

${ }^{3}$ Transplantation Surgery, C.S. Mott Children's Hospital, University of Michigan Medical Center, Ann Arbor, MI, USA

Key words: kidney transplant - bone marrow transplant - end-stage renal disease - bone marrow transplant nephropathy

Susan E. Thomas MD, Medical Director, Pediatric Kidney Transplant Program, Division of Pediatric Nephrology, C.S. Mott Children's Hospital, University of Michigan Medical Center, F6865/Box 0297, Ann Arbor, Ml 48109, USA.

Tel.: 7349364210

Fax: 7347636997

E-mail: sethomas@umich.edu

Accepted for publication 29 January 2004

Abbreviations: BMT, bone marrow transplant; CCSG, Children's Cancer Study Group; CDDP, cisplatin; CT, computed tomography; DR, human leukocyte antigen - class II; ESRD, end-stage renal disease; GI, gastrointestinal; GVHD, graft-vs.host disease; HLA, human leukocyte antigen; HSCT, hematopoietic stem cell transplant; IVC, inferior vena cava; PTLD, post-transplant lymphoproliferative disease; TBI, total body irradiation. 
The first successful allogeneic BMTs in humans were carried out in 1968. Since then, use of allogeneic or autologous HSCT has increased dramatically, with an estimated 40-50 000 HSCTs worldwide in 2001(1). Long-term patient survival has also increased owing to effective pretransplant regimens, improvement in prophylaxis of GVHD, and the overall advances in supportive care (2). With increasing survival after BMT, the number of patients suffering from organ damage is also rising. BMT nephropathy has been described as the development of chronic renal failure, azotemia, hypertension and disproportionate anemia, occurring 100 days or more after BMT in the absence of known nephrotoxins (3). Examination of renal biopsy specimens via light microscopy demonstrates mesangial and endothelial cell dropout with widening of glomerular capillary loops. Electron microscopy reveals subendothelial expansion of the glomerular basement membrane $(3,4)$. BMT nephropathy affects between 0.8 and $9.5 \%$ of adults and as many as $54 \%$ of children who undergo BMT (5). Slowing progression of BMT nephropathy may be possible with angiotensin-converting enzyme inhibitors or angiotensin II receptor blockers, however progression to ESRD can occur (6). Patients who develop ESRD after BMT have a significantly decreased survival as compared with other causes of ESRD (6). Risk factors for the development of acute renal failure following BMT include: pre-existing renal impairment, toxicity of conditioning regimens, marrow infusion toxicity, nephrotoxic medications, impaired renal function closely associated with liver impairment, thrombotic thrombocytopenic purpura, infections, and acute GVHD (7). The predictive value of transient ARF for renal function in long-term survivors remains controversial. In the report of Van Why et al. (8), renal impairment during the early phase following HSCT was not predictive for later renal impairment. In contrast, Kist-van Holthe et al. found chronic renal insufficiency $1 \mathrm{yr}$ after BMT correlated with a high serum creatinine within 3 months of BMT (9). Favorable outcomes of renal transplantation following adult BMT have been reported (2, 10-13), however, outcomes in pediatric recipients have not been well described. Because of this lack of information regarding outcomes of pediatric patients undergoing renal transplantation after prior BMT, we analyzed our single center experience.

\section{Patients and methods}

Case 1

AA presented at $2 \mathrm{yr}$ of age with vomiting, abdominal distension, and IVC obstruction. A CT scan revealed a large abdominal mass displacing the right kidney across the midline. The diagnosis of stage III neuroblastoma was made. AA received emergent radiation secondary to IVC obstruction (1800 cGy), was treated with the CCSG 321-P2 protocol, and underwent surgery to remove the right adrenal tumor. Later the same year she received pre-BMT conditioning with Cisplatin (CDDP) Teniposide (VM-26), melphalan, and TBI (1000 cGy) in preparation for allogeneic BMT. She received a BMT at the University of Minnesota from an HLA identical brother. Serum creatinine at the time of BMT ranged from 0.9 to $1.2 \mathrm{mg} / \mathrm{dL}$. Over the next 10 yr AA's post-BMT course was complicated by CMV pneumonitis, delayed puberty, persistent short stature secondary to ovarian failure, hypothyroidism, cardiomyopathy, failure to thrive requiring G-tube placement, and progressive chronic renal failure with tubular electrolyte wasting. At $12 \mathrm{yr}$ of age AA received a preemptive renal transplant from her aunt (one DR match) at the University of Michigan. The bone marrow donor was $<18 \mathrm{yr}$ of age, and therefore did not serve as the patient's kidney donor. No induction therapy was given. She was initially treated with cyclosporine, prednisone and mycophenolate mofetil, but was converted to tacrolimus secondary to gingival hyperplasia and hirsuitism. At $16 \mathrm{yr}$ of age she was diagnosed with basal cell carcinoma of her forehead and underwent wide local excision with a full-thickness skin graft. AA is currently $6 \mathrm{yr}$ status post-renal transplant and has a creatinine of $0.7 \mathrm{mg} / \mathrm{dL}$ (Table 1 ).

\section{Case 2}

$\mathrm{BB}$ is the product of a $34 \mathrm{wk}$ gestation born to a 29 -yr-old healthy woman $G_{2} P_{1}$ without known teratogen exposures (14). He exhibited growth retardation, bilateral cryptorchidism, and crossed fused ectopic right-sided kidney (without renal impairment) but was otherwise healthy. At 4 yr of age, he developed proteinuria that progressed to nephrotic syndrome. An open renal biopsy demonstrated minimal focal and segmental mesangial proliferation and $\mathrm{IgG}, \operatorname{IgA}, \mathrm{IgM}$, C3 and C1Q deposition consistent with immune complex nephritis. Angiotensin-converting enzyme inhibitor therapy was begun to decrease proteinuria. At $4.25 \mathrm{yr}$ of age he was noted to have persistent neutopenia (absolute neutrophil count of $874 / \mathrm{mm}^{3}$ ). His bone marrow displayed $30 \%$ trilineage hypocellularity, and results of investigations for bone marrow syndromes were negative. He developed multiple systemic infections, prompting a G-CSF trial, which normalized his absolute neutrophil count. Lymphopenia with T-cell depletion (CD3, CD4, and CD8 deficiencies) and spared B-cell function progressed. These findings along with mild dysmorphic features and radiographic evidence of spondyloepiphyseal dysplasia suggested the diagnosis of Schimke immuno-osseous dysplasia. At $6 \mathrm{yr}$ of age, BB underwent an allogeneic BMT (HLA identical brother) at the University of Michigan. His conditioning therapy consisted of oral busulfan, and intravenous cyclophosphamide. GVHD prophylaxis consisted of intravenous tacrolimus and methotrexate. Engraftment occurred (absolute neutrophil count $>500 / \mathrm{mm}^{3}$ ) on day 12, but acute GVHD (grade III skin, grade IV GI) developed on day 26. 


\section{Renal transplantation following prior bone marrow transplantation}

Table 1. Patient data

\begin{tabular}{|c|c|c|c|}
\hline Primary disease & Neuroblastoma (III) & Schimke's immuno-osseous dysplasia & Neuroblastoma (IV) \\
\hline Prior chemo/XRT & Yes/yes & Yes/no & Yes/yes \\
\hline Renal disease & Interstitial fibrosis & Mesangioproliferative GN & Interstitial fibrosis \\
\hline Age at BMT & $2 \mathrm{yr}$ & $7 \mathrm{yr}$ & $4 \mathrm{yr}$ \\
\hline Age at renal tx & $12 \mathrm{yr}$ & $8 \mathrm{yr}$ & $14 \mathrm{yr}$ \\
\hline Source of BMT & Brother (HLA identical) & Brother (HLA identical) & Autologous \\
\hline Source of renal tx & Aunt & Father & Father \\
\hline GVH pre/post renal tx & No/No & Yes (Skin III, GI IV)/yes (GI I) & No/no \\
\hline PRA (historic/time of renal tx) & $0 / 0$ & $0 / 0$ & $0 / 0$ \\
\hline Renal tx HLA match & $1 \mathrm{DR}$ & 1 Haplotype & 1 Haplotype \\
\hline Induction immunosuppression & None & Simulect & Simulect \\
\hline Current immunosuppression & FK/Pred/MMF* & FK/Pred ${ }^{*}$ & FK/Pred/MMF* \\
\hline Biopsy proven rejection & No & No & No \\
\hline Current creatinine $(\mathrm{mg} / \mathrm{dL})$ & 0.7 & 0.6 & 1.2 \\
\hline Current WBC (K/MM3) & 5.6 & 8.7 & 8.3 \\
\hline Current HCT (\%) & 39 & 39 & 36 \\
\hline Current PIts (K/MM3) & 232 & 229 & 111 \\
\hline Hypertension & Yes & Yes & Yes \\
\hline Diabetes mellitus & No & No & No \\
\hline Hyperlipidemia & No & No & No \\
\hline Opportunistic infection & No & Adenoviral enteritis $\times 1$ & No \\
\hline Recurrence of primary malignancy & No & NA & No \\
\hline Post-transplant malignancy & Basal cell $\mathrm{CA} \times 1$ & No & No \\
\hline Current graft survival & $6 \mathrm{yr}$ & 3 yr 8 months & 7 months \\
\hline
\end{tabular}

${ }^{*} F K$, tacrolimus; Pred, prednisone; MMF, mycophenolate mofetil.

His GI-GVHD failed to respond to methylprednisolone and intravenous mycophenolate, and he did not tolerate antithymocyte globulin. Because of progressive GI-GVHD, anti-CD2 monoclonal antibody therapy was given and led to resolution of GI-GVHD. Intermittant anorexia, nausea, and vomiting required transpyloric gastrojejunal tube feedings. His post-BMT course was further complicated by malignant hypertension, decreased bone maturation, impaired growth and diabetes mellitus. He developed progressive nephrotic syndrome requiring albumin infusions. By 7 months after BMT, he required hemodialysis. At 20 months following BMT, he received a kidney from his father (1 haplotype) at the University of Michigan. The bone marrow donor was $<18 \mathrm{yr}$ of age, and therefore did not serve as the patient's kidney donor. BB received Simulect ${ }^{\circledR}$ (Novartis Pharmaceuticals Corporation, East Hanover, NJ, USA) induction and was initiated on cyclosporine, prednisone, and mycophenolate mofetil immunosuppression. He was later converted to tacrolimus and prednisone because of GI intolerance of mycophenolate. BB is now $3 \mathrm{yr}$ 8 months status post-living related renal transplant with full hematologic engraftment, and stable renal transplant function (Table 1). He experienced a Grade I GI GVHD flare 6 months following renal transplant, and an episode of adenoviral gastroenteritis 22 months following renal transplant. His serum creatinine is stable at $0.6 \mathrm{mg} / \mathrm{dL}$, and he has had no post-transplant malignancies to date.

\section{Case 3}

CC was diagnosed at 18 months of age with stage IV neuroblastoma after presenting with an abdominal mass. He was treated with CCSG protocol 321-P2, (2100 cGY) of radiation to the tumor and debulking surgery. He experienced a relapse of his neuroblastoma at $3 \mathrm{yr} 8$ months of age. At the time of his relapse, $\mathrm{CC}$ could not receive standard melphalan and TBI because of his prior radiation therapy. Therefore he was treated with busulfan, carboplatin, and thiotepa. CC underwent autologous BMT at $4 \mathrm{yr}$ of age at Harker Hospital. Serum creatinine following BMT was $0.8 \mathrm{mg} / \mathrm{dL}$. Following BMT, CC experienced veno-occlusive disease of the liver, esophageal varices, seizure disorder, short stature, failure to thrive, and progressive chronic renal failure with tubular dysfunction. At $14 \mathrm{yr}$ of age $\mathrm{CC}$ received a preemptive renal transplant from his father (one haplotype) at the University of Michigan. He received Simulect ${ }^{\circledR}$ induction and tacrolimus, prednisone, and mycophenolate mofetil maintenance immunosuppression. CC experienced transient hyperglycemia within the first 30 days following renal transplant but did not require insulin therapy. He underwent a percutaneous renal transplant biopsy 6 months post-renal transplant for a serum creatinine of $1.4 \mathrm{mg} / \mathrm{dL}$. Biopsy findings were consistent with tacrolimus toxicity, and his creatinine has returned to baseline $(1.2 \mathrm{mg} / \mathrm{dL})$ with a reduction in his tacrolimus dose. BB is currently 7 months following renal transplant and has been without opportunistic infections or secondary malignancies (Table 1).

\section{Discussion}

Over the years there have been numerous advances that have led to improved outcomes and quality of life for pediatric patients with ESRD. Refined surgical techniques, improved immunosuppressive protocols, and better management of children before and after transplantation have led to superior patient and graft outcomes following pediatric renal transplantation. The pediatric renal transplant community is now challenged with caring for a new patient population, the pediatric bone marrow recipient with 
ESRD. These children have been exposed to past chemotherapy, irradiation, and immunosuppression. However, they may also present with preexisting GVHD, cardiovascular disease, bone disease, diabetes mellitus, gonadal failure, short stature, sequelae of past opportunistic infections, increased risk of developing recurrence of their primary malignancy, and increased risk of developing a secondary malignancy. Therefore before proceeding with renal transplantation, the potential benefit of renal transplantation should be weighed against the possible risk of malignancy and other complications (13). Curtis et al. studied 19229 patients who received allogeneic BMTs $(97.2 \%)$ or syngeneic BMTs $(2.8 \%)$ between 1964 and 1992 at 235 centers to evaluate the risk of the development of a new solid cancer (15). The risk was significantly elevated for malignant melanoma, cancers of the buccal cavity, liver, brain or other parts of the central nervous system, thyroid, bone, and connective tissue. The risk was higher for recipients who were younger at the time of transplantation. In multivariate analyses, higher doses of total-body irradiation were associated with a higher risk of solid cancers. Chronic GVHD and male sex were strongly linked with an excess risk of squamouscell cancers of the buccal cavity and skin (15).

Solid organs contain immunologically significant 'passenger lymphocytes' (11). These cells of donor origin migrate and widely populate the tissues of the recipients, potentially resulting in a state of microchimerism, which has been hypothesized to be potentially tolerogenic as well as immunogenic. These donor-derived white cells and antigen presenting cells may initiate GVHD and, theoretically might influence the host's immune system and allow grafts to be accepted long-term with reduced immunosuppression (immunologic tolerance) (11). Sayegh et al. (10), have demonstrated that persons who have successful BMT and who develop ESRD may be specifically tolerant and may safely receive a kidney from the same donor without immunosuppression. These findings have subsequently been confirmed $(3,12,13,16,17)$. It has been stressed that recipients should be carefully followed for symptoms and signs of GVHD (10) (Table 2).
In considering post-BMT patients for renal transplant, it is important to consider the relationship of the recipient, the BMT donor, and the renal transplant donor. As has been well demonstrated, the need for immunosuppression postrenal transplant is totally obviated when the bone marrow and the kidney donor are from the same individual (Table 2, scenario I). What is less clear, are the relative risks for malignancy, infection, and other immunosuppressive related morbidities in each of these three scenarios. One could speculate that there will be issues that arise which are unique for each situation. While some of these differences may reflect the conditioning regimen prior to allogeneic BMT compared with autologous BMT, it is possible that a recipient of two allogeneic transplants from two unique individuals (scenario III) is biologically distinct from the recipient of a renal transplant postautologous BMT (scenario II). If this hypothesis is true, one group of patients may benefit from different immunosuppressive strategies postrenal transplant.

Two recent reports have examined adult BMT recipients who have gone on to undergo renal transplantation. In the first by Hamawi et al. (2), kidney transplantation was performed in 10 pts with BMT nephropathy. In six patients, the kidney donor was the BMT donor. These individuals were given no long-term immunosuppression. Four other patients received kidney transplants from donors who were not the marrow donor (two living donors, two cadaveric donors). After a median follow-up of 34 months, no patient had an episode of acute rejection. All graft losses $(n=4)$ resulted from patient death. Three deaths were due to infectious processes (including two infectious deaths in patients not on immunosuppression), one from myocardial infarction/PTLD. Median estimated actuarial patient and graft survival (Kaplan-Meier) was 105 months (2). In a second single center study by Butcher et al. (13) six patients with ESRD following BMT underwent renal transplantation. In three recipients the bone marrow and kidney were from the same donor. They were managed without any immunosuppressive therapy. The patients were followed up for 31 months

Table 2. Immunosuppression requirement following renal transplant in BMT recipients

\begin{tabular}{|c|c|c|c|}
\hline Scenario & Source of BMT & Source of renal transplant & Maintenance immunosuppression \\
\hline Scenario I & Allogeneic BMT Tx donor A & Allogeneic renal Tx donor $A$ & Not required \\
\hline Scenario ॥ & Autologous BMT & Allogeneic renal Tx & Required \\
\hline Scenario III & Allogeneic BMT donor A & Allogeneic renal Tx donor B & Required \\
\hline
\end{tabular}


(3-31 months) and five of six were alive with functioning bone marrow and renal transplants. One patient who did receive immunosuppression died from metastatic squamous cell cancer of the genital tract (13).

We present three pediatric patients with a history of BMT and went on to develop ESRD requiring renal transplantation. All three patients had stable bone marrow function and were without evidence of active GVHD at the time of renal transplantation. Two of the three patients had a primary diagnosis of neuroblastoma. In a retrospective study by Lane et al. (18), 30 pediatric BMT recipients requiring dialysis for acute renal failure early after BMT were retrospectively evaluated. Patients requiring dialysis were not significantly different from the general pediatric BMT population except for: a greater proportion of neuroblastoma patients in the dialysis group, and that there were fewer autologous and more unrelated BMT donors in the dialysis group (18). It was postulated that renal functional reserve might have been reduced in these patients by previous aggressive chemotherapy, tumor debulking procedures which sometimes necessitated nephrectomy, or prior abdominal irradiation (18).

Our two BMT recipients with prior neuroblastoma developed chronic renal failure that over a 10 -yr period progressed to ESRD secondary to BMT nephropathy. Although several factors may contribute to the onset of BMT nephropathy, the underlying pathophysiology can be attributed to endothelial damage and coagulation abnormalities $(7,19-22)$. Other chronic renal diseases have been reported occasionally. Membranous nephropathy has been found in a number of adult patients after HSCT (23-29) as has minimal change nephropathy (30) and focal segmental glomerulosclerosis $(7,31)$.

In summary, renal transplantation is an excellent option for pediatric patients with ESRD following BMT. Careful attention needs to be paid to bone marrow function and pre-existing immune system impairment when weighing different immunosuppressive agents for renal transplantation. These pediatric patients need vigilant medical surveillance of multiple organ systems following their bone marrow and kidney transplants, with particular focus on opportunistic infection and malignancy. Larger scale outcome analyses will provide further insight into the long-term prognosis of patients who undergo BMT and renal transplantation. Additional efforts are required to develop optimal immunosuppressive regimens for the combined BMT/ kidney recipient. Finally, newer reno-protective strategies will be vitally important to reduce the incidence of chronic kidney disease and progression to ESRD in the BMT population in the future.

\section{Acknowledgments}

We would like to acknowledge Dr Timothy E. Bunchman, Dr Valerie P. Castle, Dr Aileen Sedman, Dr Charles A. Main, Catherine Miller RN MS CNP, and Joan Daniels $\mathrm{RD}$ for the care they provided these three patients.

\section{References}

1. International Bone Marrow Transplant Registry/Autologous Blood \& Marrow Transplant Registry. Report on state of the art in blood and marrow transplantation. IBMTR/ABMTR Newsletter 2002: 9: 1-12.

2. Hamawi K, de Magalhaes-Silverman M, Bertolatus JA. Outcomes of renal transplantation following bone marrow transplantation. Am J Transplant 2003: 3: 301-305.

3. Cohen eP, lawton CA, Moulder Je, Becker CG, Ash RC. Clinical course of late-onset bone marrow transplant nephropathy. Nephron 1993: 64: 626-635.

4. Antignac C, Gubler MC, Leverger G, Broyer $M$, HABIB R. Delayed renal failure with extensive mesangiolysis following bone marrow transplantation. Kidney Int 1989: 35: 1336-1344

5. Cohen EP, Lawton CA, Moulder JE, Bone marrow transplant nephropathy: radiation nephritis revisited. Nephron 1995: 70: 217-222.

6. Cohen EP, Piering WF, Kabler-Babbitt C, Moulder JE. End-stage renal disease (ESRD) after bone marrow transplantation: poor survival compared to other causes of ESRD. Nephron 1998: 79: 408-412.

7. Patzer L, Kentouche K, Ringelmann F, Misselwitz J. Renal function following hematological stem cell transplantation in childhood. Pediatr Nephrol 2003: 18: 623-635.

8. Van Why SK, Freidman AL, Wei LJ, Hong R. Renal insufficiency after bone marrow transplantation in children. Bone Marrow Transplant 1991: 7: 383-388.

9. Kist-van Holthe JE, Goedvolk CA, Brand R, et al. Prospective study of renal insufficiency after bone marrow transplantation. Pediatr Nephrol 2002: 17: 1032-1037.

10. Sayegh MH, Fine NA, Smith JL, Rennke HG, Milford EL, TILNEY NL. Immunologic tolerance to renal allografts after bone marrow transplants from the same donors. Ann Intern Med 1991: 114: 954-955.

11. Dey B, Sykes M, Spitzer T. Outcomes of recipients of both bone marrow and solid organ transplants: a review. Medicine (Baltimore) 1998: 77: 355-369.

12. Helg C, Chapius B, Bolle JF, et al. Renal transplantation without immunosuppression in a host with tolerance induced by allogeneic bone marrow transplantation. Transplantation 1994: 58: 1320-1421.

13. Butcher JA, Hariharan S, Adams MB, Johnson CP, RozA AM, CoHen EP. Renal transplantation for end-stage renal disease following bone marrow transplantation: a report of six cases, with and without immunosuppression. Clin Transplant 1999: 13: 330-335.

14. Petty EM, Yanick GA, Hutchinson RJ, et al. Successful bone marrow transplantation in a patient with Schimke immuno-osseous dysplasia. J Pediatr 2000: 137: 882-886.

15. Curtis RE, Rowlings PA, Deeg HJ, et al. Solid cancers after bone marrow transplantation. N Engl J Med 1997: 336: 897-904. 


\section{Thomas et al.}

16. Sellers MT, Deierhoi MH, Curtis JJ, et al. Tolerance in renal transplantation after allogeneic bone marrow transplantation-6 year follow-up. Transplantation 2001: 71: 1681-1683.

17. Gajewsi Jl, Ippoliti C, Ma Y, Champlin R. Discontinuation of immunosuppression for prevention of kidney graft rejection after receiving a bone marrow transplant from the same HLA identical sibling donor. Am J Hematol 2002: 71: 311-313.

18. Lane PH, Mauer SM, Blazar BR, Ramsay NKC, Kashtan CE. Outcome of dialysis for acute renal failure in pediatric bone marrow transplant patients. Bone Marrow Transplant 1994: 13: 613-617.

19. Natazuka T, Kajimoto K, Ogawa R, et al. Coagulation abnormalities and thrombotic microangiopathy following bone marrow transplantation from HLA-matched unrelated donors in patients with hematological malignancies. Bone Marrow Transplant 1998: 21: 815-819.

20. Kanamori H, Maruta A, SAsaki S, et al. Diagnostic value of hemostatic parameters in bone marrow transplant-associated thrombotic microangiopathy. Bone Marrow Transplant 1998: 21: 705-709.

21. Jaenke RS, Robbins MEC, Bywaters T, Witehouse E, Rezvani M, Hopewell JW. Capillary endothelium-target site of renal radiation-injury. Lab Invest 1993: 68: 396-405.

22. Monke JL, B J, Thrombotic microangiopathies associated with drugs and bone marrow transplantation. Hematol Oncol Clin North Am 1996: 10: 485-497.

23. Lin J, Markowtiz GS, Micolaides M, et al. Membranous glomerulopathy associated with graft-versus-host disease following allogeneic stem cell transplantation-report of 2 cases and review of the literature. Am J Nephrol 2001: 21: 351-356.
24. Yorioka N, TANigychi Y, Shimote K, et al. Membranous nephropathy with chronic graft-versus-host disease in a bone marrow transplant recipient (letter). Nephron 1998: 80: 371372.

25. Sato N, Kishi K, Yagisawa K, et al. Nephrotic syndrome in a bone marrow transplant recipient with chronic graft versus host disease. Bone Marrow Transplant 1995: 16: 303-305.

26. Barbara JAJ, Thomas AC, Smith PS, Gillis D, Ho JOK, WoOdRoffe AJ. Membranous nephropathy with graft-versushost disease in a bone marrow transplant recipient. Clin Nephrol 1992: 37: 115-118.

27. Muller GA, Mueller CA, Markoviclipkowski J, et al. Membranous nephropathy after bone marrow transplantation in cyclosporine treatment. Nephron 1989: 51: 555-556.

28. Rossi L, Cardarelli F, Vampa ML, Buzio C, Olivetti G. Membranous glomerulonephritis after haematopoietic cell transplantation for multiple myeloma. Nephron 2001: 88: 260-263.

29. Nergizoglu G, Keven K, Ates K, et al. Chronic graft-versushost disease complicated by membranous glomerulonephritis. Nephrol Dial Transplant 1999: 14: 2461-2462.

30. Walker JV, Morich D, Anasetti C. Minimal-change nephrotic syndorme after cyclosporine withdrawal in a marrow transplant recipient. Am J Kidney Dis 1995: 26: 532-534.

31. Oliveira JSR, Bahia D, Franco M, Balda C, Stella S, KeRBAUY J. Nephrotic syndrome as a clinical manifestation of graft-versus-host disease (GVHD) in a marrow transplant recipient after cyclosporine withdrawal. Bone Marrow Transplant 1999: 23: 99-101. 